\title{
Estudio anatómico del glande del cuy (Cavia porcellus) de la raza Perú
}

\author{
Anatomical study of the guinea pig glans (Cavia porcellus) of the Peru breed \\ Nattaly Márquez F. ${ }^{1}$, Roberto Valencia L. $(\dagger)^{1}$, Lili Chauca F. ${ }^{2}$, Gemma Verde Z. ${ }^{1,3}$
}

\section{Resumen}

El presente estudio tuvo como objetivo describir las características macroscópicas y microscópicas de las estructuras que conforman el glande del pene del cuy y sus modificaciones influenciadas por la edad. El estudio se realizó en 24 cuyes distribuidos en cuatro grupos etarios (1, 2, 3, 5 meses) de la raza Perú. Se utilizaron 20 muestras para el estudio macroscópico y cuatro muestras para el estudio microscópico. La superficie externa del glande presentó escamas córneas pequeñas, de borde convexo, acompañado de diminutas espículas dirigidas en craneal. En la superficie dorsal y ventrolateral se observó la presencia de escamas desarrolladas de forma alargada con el borde libre convexo y con numerosas espículas dirigidas en sentido dorsocraneal. El saco del glande se situó dorsal a la uretra peneana y la superficie interna poblada de pequeñas escamas y pliegues longitudinales. El saco del glande contiene en su interior un par de procesos estiloides, de naturaleza córnea que se originan en su base. El hueso del pene es de forma alargada y se relaciona a la superficie ventral del glande. El glande presentó un epitelio de transición, que asienta en tejido conectivo laxo vascularizado. Los procesos estiloides al corte transversal presentaron un grueso estrato córneo compuesto por capas de queratina, y el os penis se encuentra formado por tejido óseo esponjoso. Con el avance de la edad los extremos de los procesos estiloides se agudizan, se vuelven divergentes y en algunos casos asimétricos.

Palabras clave: cuy; glande; escamas; espículas; saco del glande; procesos estiloides; os penis

\footnotetext{
${ }^{1}$ Facultad de Medicina Veterinaria y Zootecnia, Universidad Peruana Cayetano Heredia, Lima, Perú

2 Instituto Nacional de Innovación Agraria - INIA, Lima, Perú

${ }^{3}$ E-mail: gemma.verde.z@upch
}

Recibido: 10 de julio de 2019

Aceptado para publicación: 15 de agosto de 2019 
The purpose of the study was to describe the macroscopic and microscopic characteristics of the structures that conforms the penis glans of the Peruvian guinea pig, and changes in the structure related to animal age. The study was conducted in 24 guinea pigs distributed in four age groups (1,2, 3, 5 months) of the Peru breed, where 20 samples taken for the macroscopic study and four for the microscopic study. The glans external surface is covered by small corneous scales of convex edge with tiny spicules directed to cranial. In the dorsal and ventral-lateral surfaces is observed the presence of extended and well-developed scales that are convex and have a free edge, with spicules directed dorsal-cranial. The glans sac is located dorsal to the penile urethra and the internal wall is covered by small scales and longitudinal folds and contains a pair of keratinaceous styluses. The os penis has an extended shape and related to the ventral surface of the glans. The glans showed a transition epithelium and lax vascular tissue. The stylus processes have a thick corneous layer of keratin and the os penis is formed of spongy bone tissue. The free end of the stylus processes becomes sharper, divergent and in some cases asymmetric with age.

Key words: guinea pig; glans; scales, spicules; sac of glans; keratinaceous styles; os penis

\section{INTRODUCCIÓN}

El cuy (Cavia porcellus), conocido también como cobayo, curi, conejillo de indias, así como guinea pig en países de habla inglesa, es un mamífero roedor originario de la zona andina de Perú, Bolivia y Ecuador (Chauca, 1997a,b). A nivel mundial, es una especie que es usada como mascota y comúnmente se viene empleando como un modelo roedor para investigaciones, específicamente en el área biomédica. El cuy, en comparación con otros modelos roedores se acerca más al modelo humano, por el metabolismo de la vitamina $\mathrm{C}$ y por su respuesta inmunológica (Fox et al., 2015).

Los cuyes habitan desde las zonas bajas de la costa hasta alturas de $4500 \mathrm{msnm}$, tanto en zonas cálidas como frías (Chauca, 1997b). Se estima una población de 35 millones de cuyes en los países andinos, de los cuales 22 millones se encuentran en el Perú, país que cuenta con más de 700 mil unidades agropecuarias dedicadas a su crianza. El Perú produce 16500 t anuales de carne de cuy, los cuales son criados, básicamente, en sistemas de producción familiar (Chauca, 1997a; INEI, 2012).

En el Perú se han desarrollado razas y líneas de cuyes que han mejorado sus índices productivos y reproductivos. Entre ellas, la raza Perú se caracteriza principalmente por una mayor ganancia de peso y con un tamaño de camada de 2.6 crías, en tanto que la raza Andina tiene un tamaño de camada de 3.6 crías (INIA, 2013). Los machos pueden realizar el primer empadre a los 4 meses, edad que han obtenido un tamaño adecuado, madurez sexual y peso aproximado de $1.1 \mathrm{~kg}$ (Chauca, 1997a). Así mismo, la selección de los reproductores machos debe hacerse desde los 3 meses para probarlos antes del empadre (Chauca, 1997b; Hafez E y Hafez B, 2000).

El macho posee glándulas sexuales accesorias como las vesículas seminales, próstata, glándulas coagulantes y glándulas bulbo uretrales. Los testículos se ubican en la zona inguinal y el glande es de tipo rudimentario (Valencia,1978; Vásquez y Del Sol, 2010; Wagnner y Manning, 2014; Fox et al., 2015). 
Stan (2015) reconoce dos partes en el pene de los cuyes: el cuerpo del pene y el glande, ambos con similar diámetro. Así mismo, describió la S peniana e identificó prominencias, como pequeñas espículas, a lo largo del glande, algunas con aspecto discontinuo en la región dorsal y con disposición más continua en la región ventral. La presencia de las espículas favorece la estimulación de la ovulación espontánea en las hembras (Cooper y Schiller, 1975; Noonan, 1994).

La conformación anatómica y la función de las diversas estructuras que conforman el glande del cuy no están completamente estudiadas, desconociéndose su grado de participación en la cópula. El presente trabajo tuvo por finalidad sentar las bases anatómicas para estudios aplicados relacionados con la reproducción del cuy.

\section{Materiales y Métodos}

Los animales se obtuvieron del Instituto Nacional de Investigación y Extensión Agraria (INIA), entre los meses de enero a junio de 2006. La disección y el procesamiento histológico de las muestras se realizaron en el Laboratorio de Anatomía y en el Laboratorio de Patología, respectivamente, de la Facultad de Veterinaria y Zootecnia de la Universidad Peruana Cayetano Heredia, Lima. Los procedimientos realizados fueron aprobados por el Comité de Ética para el Uso de Animales de la Universidad Peruana Cayetano Heredia.

Se utilizaron 24 glandes de cuyes de la raza Perú PPC 0.75, distribuidas en 4 grupos etarios: 1, 2, 3 y 5 meses, de 6 animales cada uno. Los cuyes de 1 y 2 meses fueron sacrificados utilizando T61 ${ }^{\circledR}$ (mebezonio ioduro) (Intervet) por vía intrapulmonar, caudal a la escápula y tercio dorsal del tórax, en dosis de $0.1 \mathrm{ml} /$ animal, siguiendo las instrucciones del fabricante. Los cuyes de 3 y 5 meses se sacrificaron en la sala de beneficio de cuyes del INIA, siguiendo los procedimientos de rutina de la institución.
Veinte muestras (cinco por cada grupo etario) fueron trabajadas en fresco para el estudio descriptivo y biométrico del glande y de los procesos estiloides. Además, cuatro muestras (una por cada grupo etario) fueron fijadas en formol al 10\% para el estudio histológico, empleando hematoxilina y eosina para la observación del glande, saco del glande y formaciones córneas. Se midió la longitud y diámetro del glande, las dimensiones de las escamas, la abertura de la fisura media, así como la longitud y el diámetro del saco del glande. En los procesos estiloides se tomó la longitud, diámetro, forma, dirección del vértice, color y presencia de flexibilidad. Para las mediciones de tamaño se utilizó una regla milimetrada.

Se realizó el estudio anatómico comparado de las observaciones macroscópicas y microscópicas del glande en las edades propuestas. La Nomenclatura Anatómica empleada corresponde a la Nómina Anatómica Mundial de Anatomistas Veterinarios (ICVGAN, 2005).

\section{Resultados y Discusión}

El glande del pene (Glans penis) es de forma cilíndrica, mide aproximadamente 1.6 $\mathrm{cm}$ de longitud y $0.6 \mathrm{~cm}$ de diámetro en el adulto ( 5 meses de edad) (Cuadro 1). El glande se dirige en caudal, representa el extremo libre del órgano copulador y se encuentra ocupando la cavidad prepucial. El extremo del glande es redondeado, en la superficie dorsal se observa una fisura en sentido longitudinal y otra en sentido transversal, en donde se abre el orificio uretral externo. El saco del glande se encuentra dorsal a este último (Figura. 1).

La superficie externa del glande está poblada de pequeñas escamas córneas, de borde convexo, fino y aserrado dirigidas en craneal, lo cual coincide a lo reportado por Stan (2015), quien además menciona que la ubicación de las escamas le da un aspecto discontínuo. En la superficie dorsal se acen- 
Cuadro 1. Medidas (media \pm desviación estándar, $\mathrm{mm}$ ) del glande y procesos estiloides en cuyes raza Perú ${ }^{1}$

\begin{tabular}{llcccc}
\hline & \multicolumn{5}{c}{ Edad (meses) } \\
\cline { 2 - 6 } & & 1 & 2 & 3 & 5 \\
\hline Glande & Longitud & $8.8 \pm 2.4$ & $13 \pm 0.7$ & $15.6 \pm 3.2$ & $15.6 \pm 0.6$ \\
& Diámetro & $5.6 \pm 2.6$ & $5.8 \pm 0.5$ & $6.4 \pm 0.6$ & $6.4 \pm 0.6$ \\
Escamas & Dorsales & $0.6 \pm 0.4$ & $1.0 \pm 0.0$ & $1.5 \pm 0.5$ & $2.0 \pm 0.7$ \\
& Ventrales & $0.6 \pm 0.4$ & $2.5 \pm 1.3$ & $4.0 \pm 1.2$ & $4.0 \pm 1.0$ \\
Fisura media & Laterales & $0.8 \pm 0.5$ & $6.0 \pm 1.0$ & $6.0 \pm 1.0$ & $7.5 \pm 1.6$ \\
Saco del glande & & $2.4 \pm 0.6$ & $3.2 \pm 0.5$ & $3.4 \pm 0.9$ & $3.4 \pm 0.6$ \\
Procesos estiloides & Longitud & $3.2 \pm 0.8$ & $5.6 \pm 0.6$ & $6.6 \pm 0.9$ & $7.0 \pm 1.0$ \\
& Diámetro & $2.0 \pm 0.7$ & $3.4 \pm 0.9$ & $4.0 \pm 1.0$ & $4.4 \pm 0.6$ \\
& Longitud & $1.1 \pm 0.6$ & $3.4 \pm 0.6$ & $3.2 \pm 0.5$ & $3.6 \pm 0.9$ \\
& Diámetro & $0.9 \pm 0.2$ & $1.0 \pm 0.0$ & $1.0 \pm 0.0$ & $1.0 \pm 0.0$ \\
\hline
\end{tabular}

${ }^{1}$ Cinco muestras por grupo etario

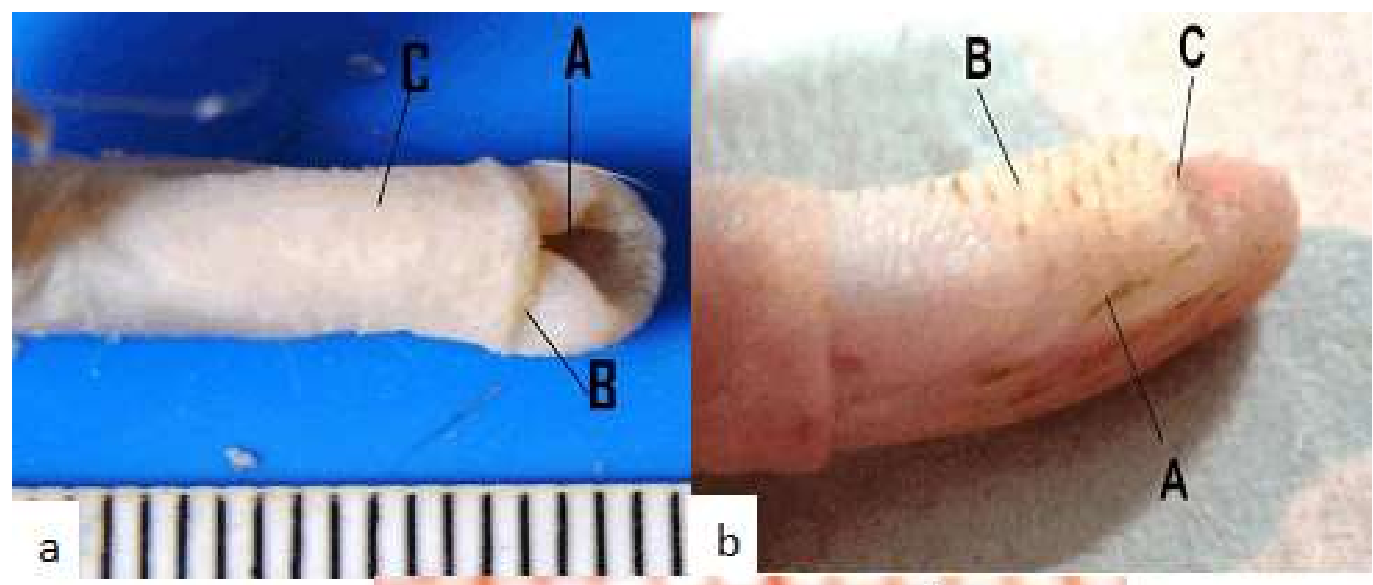

B

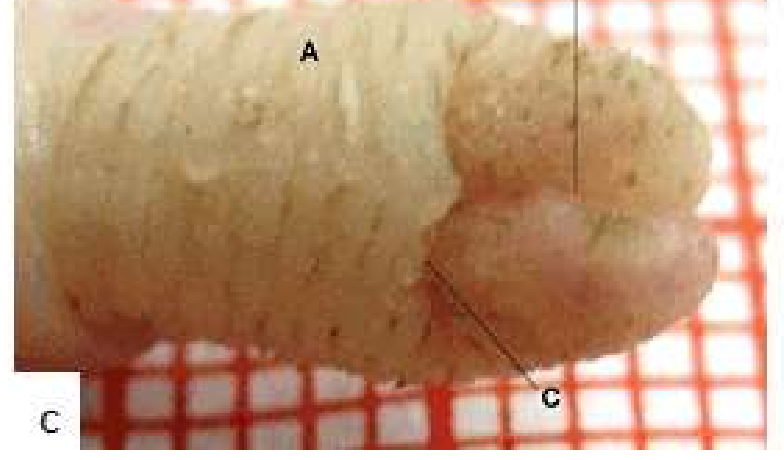

Figura 1. Vista del glande del cuy. (a) Vista dorsal. A: Fisura media, B: Fisura transversa, C: Escamas dorsales; (b) Vista lateral izquierda. A: Escamas laterales, B: Escamas dorsales, C: Fisura transversa; (c) Vista dorso-lateral. A: Escamas dorsales, B: Fisura media, C: Fisura transversa 
túa el número de escamas dispuestas en sentido transversal, con sus bordes libres dirigidos en craneal. En la superficie ventrolateral se observa la presencia de escamas desarrolladas y alargadas dispuestas longitudinalmente y paralelas entre sí, con el borde libre convexo y aserrado dirigidas en sentido dorsocraneal (Figura 1c).

La presencia del glande poblado de escamillas de diversos tamaños y disposiciones ocurre en otros roedores como la chinchilla (Chinchilla lanigera), vizcacha (Lagidium viscacia), agutí (Dasyprocta leporina) y puerco espín (Hystrix cristata) (Mollineau y Adogwa, 2006). Entre los animales domésticos que poseen este tipo de escamas se encuentra al gato (Felis catus), donde se presentan a manera de papilas córneas (König y Liebich, 2008).

El saco del glande se sitúa dorsal a la uretra peneana, presenta una pared interna de naturaleza córnea poblada de escamas pequeñas longitudinales distribuidas uniformemente. Así mismo, presenta pliegues longitudinales. En el interior del saco del glande se observa un par de procesos estiloides, de naturaleza córnea que se originan en la base del saco. Los procesos estiloides son de color blanquecino, que con el avance de la edad aumentan en longitud y diámetro (Cuadro 1). Además, los extremos de los procesos estiloides se agudizan, se vuelven divergentes y en algunos casos asimétricos (Figura $2)$. En uno de los cuyes de un mes se encontraron tres procesos estiloides de similar longitud, diámetro, forma y color. Se ha descrito que los roedores histricomorfos, como el puerco espín, agutí, chinchilla, mará, carpincho (Hydrochoerus hydrochaeris) y paca (Agouti paca) presentan el saco del glande con sus dos procesos estiloides, mientras que la vizcacha solo presenta el saco del glande (Mollineau y Adogwa, 2006). Ayala et al. (2017) mencionan que estos procesos estiloides, que los describen como espículas peneanas, influyen en la fertilidad, pero no en el comportamiento sexual y calidad espermática en esta especie.
El glande en cuyes de un mes de edad es pequeño, las escamas se encuentran en desarrollo, algunos presentan esbozos. Los procesos estiloides son de punta roma, de color blanco translúcido y son flexibles. Las escamas se encontraban en formación en dos animales y uno de ellos no presentó escama alguna. El glande en cuyes de dos y tres meses adquiere mayor tamaño, las escamas son desarrolladas, el vértice de los procesos estiloides se agudiza, adquieren mayor consistencia, y el color varía a blanco nacarado; asimismo, aumenta el tamaño de las escamas y de los procesos estiloides. En cuyes de cuatro meses, el desarrollo del glande corresponde al de un animal adulto. Las escamas y procesos estiloides aumentan ligeramente en longitud y presentan un color blanco nacarado. Los extremos libres de los procesos estiloides son alargados y con menor flexibilidad.

La luz de la uretra del pene está ocupada por la secreción de la glándula vesicular, incluso en cuyes de un mes de edad, esto corresponde al «stopper» o tapón el cual se condensa por acción de la secreción de las glándulas coagulantes, para evitar el reflujo del semen (Sofikitis at al., 1992; Lejnieks, 2007).

El promedio de longitud del pene de los cuyes adultos de la raza Perú PPC 0.75 alcanzan una longitud aproximada de $4 \mathrm{~cm}$ y un diámetro de $0.8 \mathrm{~cm}$ (Frandson, 1988). El glande ocupa la parte ventral del septo escrotal. El orificio prepucial se abre hacia atrás entre las bolsas escrotales, lo cual también ocurre en otros roedores y lagomorfos (Mollineau y Adogwa, 2006; Stan, 2015).

El pene presenta la túnica albugínea delgada, los cuerpos cavernosos y esponjoso están ligeramente desarrollados, lo cual permite una ligera erección por turgencia, tal y como lo describe Mega y Umi (2017). El proceso de erección corresponde mayormente al alargamiento con pérdida de la disposición de herradura del pene gracias a la contracción del músculo erector del pene (músculo isquiocavernoso o músculo pubo-cavernoso), 

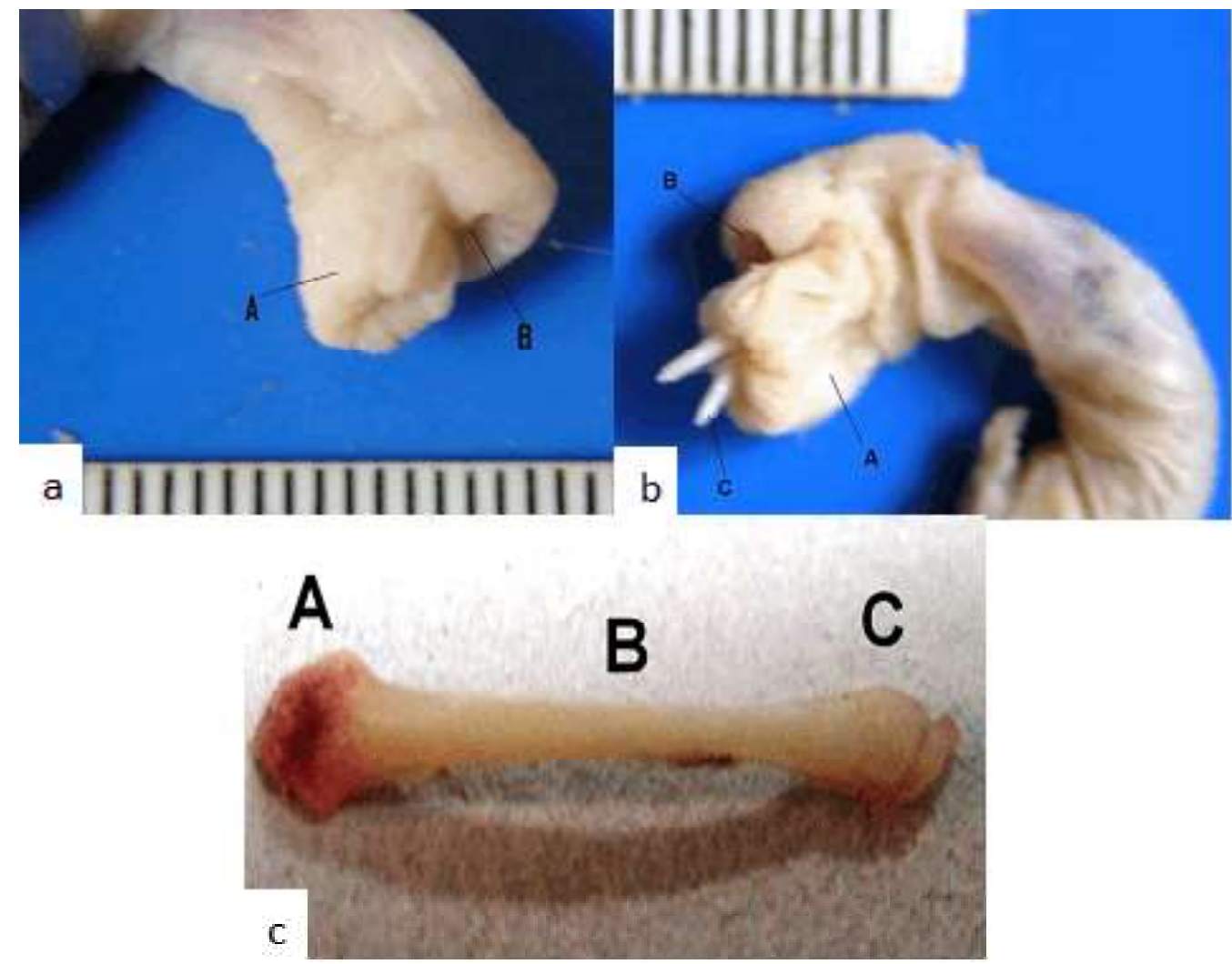

Figura 2. Vistas del glande y del os penis del pene del cuy. (a) Vista lateral derecha del glande. A: Saco del glande, B: Orificio uretral; (b) Vista lateral izquierda del glande. A: Saco del glande, B: Orificio uretral, C: Procesos estiloides; (c) Vista dorsal del os penis del pene del cuy. A: Extremidad caudal, B: Cuerpo del os penis, C: Extremidad craneal
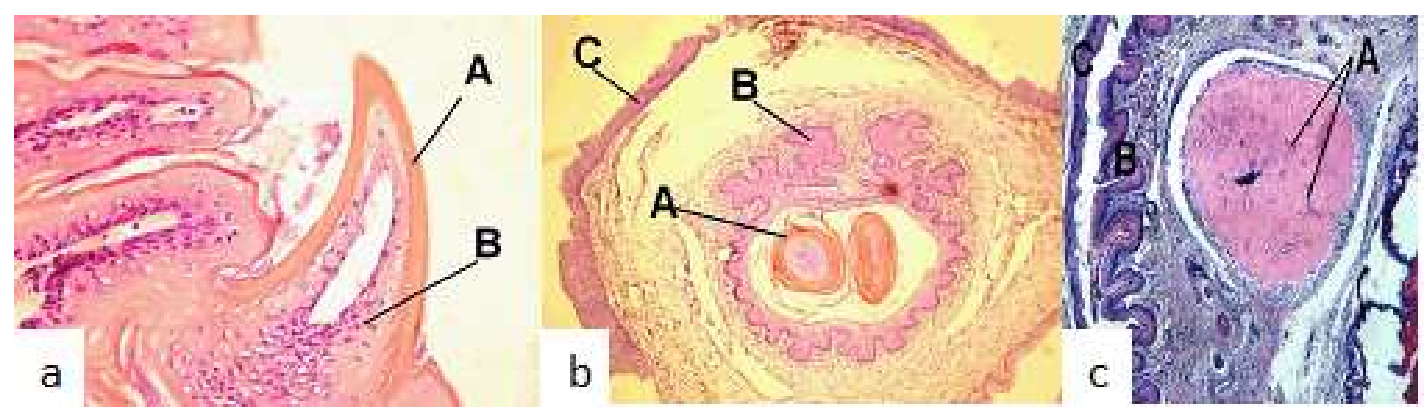

Figura 3. Corte transversal del glande, del proceso estiloide y del os penis. (a) Corte transversal del glande 10x. HE. A: Capas de queratina de los procesos estiloides, B: Epitelio de transición del saco del glande. C: Epitelio de transición de la mucosa del glande; (b) Corte longitudinal del proceso estiloide. 40x. HE. A: Capas de queratina, B: Células epiteliales; (c) Os penis. 10X. HE. A: Osteocitos y lagunas, B: Mucosa del glande, C: Mucosa del prepucio 


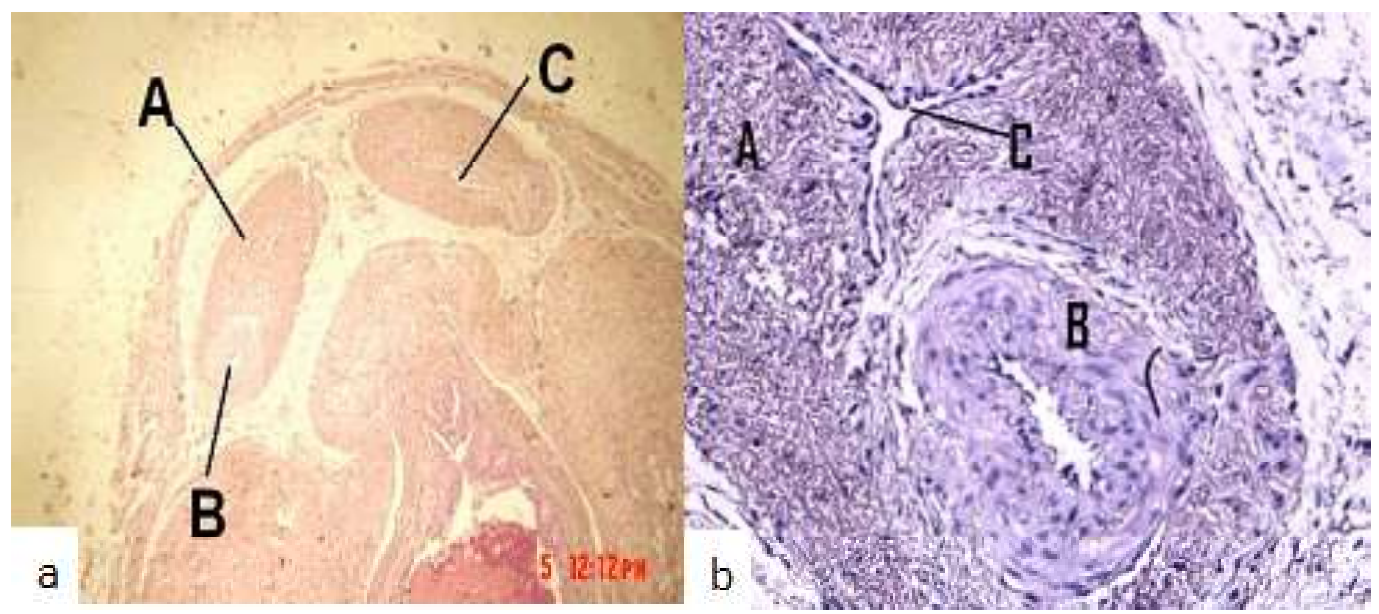

Figura 4. Cortes transversales del pene. (a) Corte transversal del pene 10X. HE. A: Tejido Conjuntivo denso regular de las cuerdas del saco del glande, B: Arteriola, C: Vénula; (b). Corte transversal del pene 40X. HE. A: Tejido Conjuntivo denso regular de las cuerdas del saco del glande, B: Arteriola, C: Vénula

el cual dirige al glande hacia delante. Un tercer mecanismo esta dado por la turgencia del glande y evaginación del saco del glande acompañado de los procesos estiloides (Cooper y Schiller, 1975; Stan, 2015).

El glande presenta un epitelio de transición no queratinizado, con células de forma ovoide, alineadas en diferente orden, que se asientan sobre un tejido conectivo laxo vascularizado. Externamente se observan crecimientos queratinizados, distribuidos irregularmente (Figura 3a). El saco del glande está tapizado internamente por un epitelio de transición, formado por células epiteliales que se proyectan a la luz del saco, y recubiertas por una capa definida de queratina. En la luz del saco se observan dos proyecciones de forma cilíndrica con vértices agudos que corresponden a los procesos estiloides. Al corte transversal del saco se observan pliegues del epitelio. En el tejido conjuntivo laxo se observa regular cantidad de arteriolas, vénulas y vasos linfáticos (Figura 3a).
Al corte transversal, el par de procesos estiloides presentan un grueso estrato córneo compuesto por capas de queratina que asienta sobre capas de células epiteliales (Figura 3b). A nivel del tercio proximal del cuerpo del pene se observan dos formaciones simétricas a manera de cuerdas del saco del glande, formadas por tejido conjuntivo denso regular, cada una con una vénula en posición medial y una arteriola en lateral. Estas se encuentran rodeadas por tejido conjuntivo laxo y más externamente por músculo liso (Figura 4). El os penis está formado por tejido óseo esponjoso y en la matriz se observan osteocitos ocupando sus respectivas lagunas (Figura 3c), lo cual coincide con Mega y Umi (2017), quienes mencionan que se observa como una estructura similar a un tejido óseo sólido.

\section{Literatura Citada}

1. Ayala L, Rodas R, Almeida A. 2017. Espículas peneanas del cobayo (Cavia porcellus), influencia sobre el compor- 
tamiento sexual, fertilidad y calidad espermática. Rev Prod Anim 29: 36-42.

2. Chauca L. 1997a. Producción de cuyes (Cavia porcellus). Roma: FAO. 77 p. [Internet]. Disponible en: http:// www.fao.org/3/W6562S/W6562S00.htm

3. Chauca L. 1997b. Producción de cuyes. Lima, Perú: INIA. 78 p.

4. Cooper G, Schiller A. 1975. Anatomy of the guinea pig. Cambridge, USA: Harvard University Press. 417 p.

5. Fox J, Anderson L, Otto G, PritchettCorning K, Whary M. 2015. Laboratory animal medicine. $3^{\text {rd }}$ ed. Elsevier: China. 1663 p.

6. Frandson RD. 1988. Anatomía y fisiología de los animales domésticos. $4^{\circ}$ ed. México: Ed Interamericana. $527 \mathrm{p}$.

7. Hafez E, Hafez B. 2002. Reproducción e inseminación artificial en animales. $7^{\circ}$ ed. México: McGraw Hill. 509 p.

8. [ICVGAN] International Committee of Veterinary Gross Anatomical Nomenclature. 2005. Nomina Anatomica Veterinaria. $5^{\text {th }}$ ed. World Association of Veterinary Anatomists. $166 \mathrm{p}$.

9. [INEI] Instituto Nacional de Estadística e Informática. 2012. IV Censo Nacional Agropecuario. [Internet]. Disponible en: http://censos.inei.gob.pe/ cenagro/tabulados/

10. [INIA] Instituto Nacional de Innovación Agraria. 2013. Cuyes. [Internet]. Disponible en: http://www.minag.gob.pe/ portal/sectoragrario/pecuaria/situacionde-las actividades-de-crianza-y-produccion/cuyes

11. König H, Liebich H. 2008. Anatomía de los animales domésticos: texto y atlas en color. Tomo II. $2^{\circ}$ ed. Madrid: Ed Médica Panamericana. 381 p.
12. Lejnieks D. 2007. Urethral plug in a rat (Rattus norvegicus). J Exotic Pet Med 16: 183-185.

13. Mega E, Umi L. 2017. Anatomy and histology of reproductive organs of male guinea pig as a source of learning. 4th International Conference on Science, Engineering the Technology. Kuala Lumpur, Malaysia

14. Mollineau W, Adogwa A. 2006. The gross anatomy of the male reproductive system of a neotropical rodent: the agouti (Dasyprota leporina). Anat Histol Embryol 35: 47-52. doi: 10.1111/j.14390264.2005.00656.x

15. Noonan D. 1994. The guinea pig (Cavia porcellus). ANZCCART News 7(3): 1-7.

16. Sofikitis N, Takahashi C, Kadowaki H, Okazaki T, Shimamoto T, Nakamura I, Miyagawa I. 1992. The role of the seminal vesicles and coagu-lating glands in fertilization in the rat. Int $\mathbf{J}$ Androl 15: 54-61. doi: 10.1111/j.13652605.1992.tb01114.x

17. Stan F. 2015. Anatomical particularities of male reproductive system of guinea pigs (Cavia porcellus). Bull UASVM Vet Med 72: 288-295. doi: 10.15835/ buasvmen-vm: 11410

18. Valencia R. 1978. Contribución al estudio anatómico del aparato reproductor macho del cuy (Cavia porcellus). Tesis de Médico Veterinario. Lima: Univ Nacional Mayor de San Marcos. 36 p.

19. Vásquez B, Del Sol M. 2010. Estudio morfológico de la próstata y glándulas vesiculares de cobayo (Cavia porcellus). Int J Morphol 28: 1301-1307. doi: 10.4067/ S0717-95022010000400050

20. Wagnner J, Manning P. 2014. The biology of the guinea pig. USA: Academic Press. 317 p. 